\title{
The effect of turbulence modelling on the assessment of platelet activation
}

\author{
Silvia Bozzi *, Davide Dominissini, Alberto Redaelli, Giuseppe Passoni \\ Politecnico di Milano, Department of Electronics Information and Bioengineering, Milano, Italy
}

\section{A R T I C L E I N F O}

\section{Keywords:}

Platelet activation

Mechanical heart valves

Turbulence

CFD models

DNS

LES

RANS

\begin{abstract}
A B S T R A C T
Pathological platelet activation by abnormal shear stresses is regarded as a main clinical complication in recipients of cardiovascular mechanical devices. In order to improve their performance computational fluid dynamics (CFD) are used to evaluate flow fields and related shear stresses. CFD models are coupled with mathematical models that describe the relation between fluid dynamics variables, and in particular shear stresses, and the platelet activation state (PAS). These models typically use a Lagrangian approach to compute the shear stresses along possible platelet trajectories. However, in the case of turbulent flow, the choice of the proper turbulence closure is still debated for both concerning its effect on shear stress calculation and Lagrangian statistics. In this study different numerical simulations of the flow through a mechanical heart valve were performed and then compared in terms of Eulerian and Lagrangian quantities: a direct numerical simulation (DNS), a large eddy simulation (LES), two Reynolds-averaged Navier-Stokes (RANS) simulations (SST $k$ - $\omega$ and RSM) and a "laminar" (no turbulence modelling) simulation. Results exhibit a large variability in the PAS assessment depending on the turbulence model adopted. "Laminar" and RSM estimates of platelet activation are about $60 \%$ below DNS, while LES is $16 \%$ less. Surprisingly, PAS estimated from the SST $k$ - $\omega$ velocity field is only $8 \%$ less than from DNS data. This appears more artificial than physical as can be inferred after comparing frequency distributions of PAS and of the different Lagrangian variables of the mechano-biological model of platelet activation. Our study indicates how much turbulence closures may affect platelet activation estimates, in comparison to an accurate DNS, when assessing blood damage in blood contacting devices.
\end{abstract}

\section{Introduction}

It is well known that mechanical cardiovascular devices can be followed by thromboembolic consequences for which anticoagulant therapy is mandatory (Ando and Yamamoto, 2009; Casa et al., 2015; Peura et al., 2012; Russell-Puleri et al., 2017). The phenomenon is triggered by blood-device interaction in terms of both chemical and mechanical stimuli (Kroll et al., 1996). The exposure to higher, more discontinuous shear stresses, than in physiological situations, is per se a sufficient condition to induce platelet activation and aggregation (Nesbitt et al., 2009; Ruggeri et al., 2006). Indeed, shear-mediated platelet activation (SMPA) is often the driving factor for thrombotic complications in presence of anatomic pathologies and cardiovascular devices (Consolo et al., 2019; Slepian et al., 2017; Yoganathan et al., 2005).

In this framework CFD techniques allow for detailed simulation of the hemodynamics through complex medical devices, such as ventricular assist devices (VAD) and mechanical heart valves (MHV). CFD tools have also been coupled with blood damage models, to evaluate the hemocompatibility of devices in terms of haemolysis and platelet. In particular, new numerical models (Nobili et al., 2008; Soares et al., 2013) have been developed to calculate the platelet activation state due to flow shear stresses. These models consist in the Lagrangian analysis of possible platelet trajectories along which some flow quantities, relevant to platelet activation, are calculated. These models were initially based on the power law equation proposed by Giersiepen et al. (1990). Later the platelet damage accumulation concept (Nobili et al., 2008) was introduced, further improved including the stress rate (Consolo et al., 2017; Soares et al., 2013). An extensive discussion on the methodological aspects of the Lagrangian computations is in Marom and Bluestein (2016).

For a thorough calculation of platelet trajectories, the accuracy of the velocity field is crucial especially in large vessels, like aorta, where typical Reynolds numbers entail a hierarchy of flow structures (Malinauskas et al., 2017; Ku, 1997). The so-called Reynolds-averaged

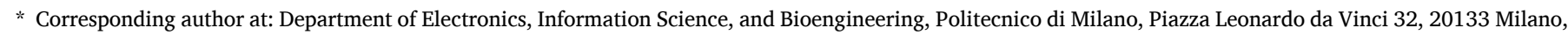
Italy.

E-mail address: silvia.bozzi@polimi.it (S. Bozzi).
} 
Navier-Stokes (RANS) simulations have been and still remain the most common approach for turbulent flows in complex geometries. Despite their long history (Prandtl et al., 1925) these are essentially based on empirical relations whose parameters require problem-dependent calibrations. The choice among different turbulence closures is still an open issue since most of them are accurate for the average flow characteristics only, while for shear stress and its derivatives this is much more uncertain, especially when calculations are cast in a Lagrangian framework (Ge et al., 2008; Goubergrits et al., 2019; Le and Sotiropoulos, 2013; Song et al., 2003). Recently, Pal et al. (2014) presented an interesting analysis, but limited to large eddy simulation (LES) closure models and to the flow variables. As a matter of fact, the effects of the different turbulence closures (RANS, LES and DNS) on the resulting Lagrangian statistics and on the platelet activation have not yet been ascertained.

In this paper we show how much turbulence models affect the flow solution around and paste a bileaflet mechanical heart valve (BMHV) and to which extent these differences map onto Lagrangian statistics and platelet activation. Five numerical simulations are presented: a direct numerical simulation, a large eddy simulation, two Reynolds-averaged Navier-Stokes simulations and a "laminar" (no turbulence modelling) simulation. These models are compared in terms of Eulerian fields (blood velocity and fluid stresses), Lagrangian quantities, estimated from the fluid stress history along the particle trajectories, and platelet activation state.

\section{Methods}

Turbulence consists in a wide spectrum of structures dynamically interacting each other and with the mean flow. Numerical methods for turbulent flow calculations can be divided into three categories, i.e. turbulence models for the Reynolds-averaged Navier-Stokes (RANS) equations, large eddy simulation (LES) and direct numerical simulation (DNS); details are in the supplementary information.

This analysis has been focused on different turbulent models with different levels of accuracy: (1) a robust isotropic RANS turbulent closure (SST $k-\omega$ ), which performs well in confined flows and in presence of bluff bodies (such as cardiac valves), (2) an anisotropic RANS model (Reynolds Stress Model, RSM), which accounts for the directional effects of the Reynolds stresses, (3) a LES model, able to resolve some finer space-time features and (4) a DNS approach which is the most accurate flow solution. Moreover, we also performed a (5) "laminar" simulation, i.e., a Navier-Stokes integration, without any turbulence closure, on a coarser grid than that used for the fully resolved DNS. This simulation gives an insight on the effects of an insufficient grid resolution, which is an issue when low Reynolds flows are solved without turbulent closures.

\subsection{Geometrical modelling}

The valve used here is the Sorin Bicarbon Fitline Size 25 (Fig. 1b), produced by Sorin (now LivaNova, Cardiac Surgery Unit). The geometrical discretization was created with ANSYS ${ }$ SpaceClaim 18.2 starting from the CAD file provided by the manufacturer. The two leaflets are set at full opening since this configuration last for a long time interval around the velocity peak (Cheng et al., 2004; Dasi et al., 2007; Le and Sotiropoulos, 2013), when turbulence originates. The small hinge mechanism was modelled intercepting two spherical caps (radius 4.5 $\mathrm{mm})$. The artificial gap size $(320 \mu \mathrm{m})$ is larger than the real one $(\approx 240$ $\mu \mathrm{m}$ ), so that 10 cells are in the gap to preserve mesh quality and minimize computational problems (Morbiducci et al., 2009).

The BMHV has been placed between two straight tubes (diameter D $=22 \mathrm{~mm}$ ) to mimic a standard mock loop set-up used in in-vitro testing of mechanical heart valves, such as the ViVitro Left Heart Simulator (ViVitro Labs Inc., Victoria, BC, Canada) (Fig. 1a). After sensitivity analysis the entry region was set equal to $2 D$ while the tube past the valve was extended to $5 D$, to have fully developed flow at the outlet. Downstream the valve, the aortic sinuses were sketched as prolate spheroids with major axis of $22 \mathrm{~mm}$, oriented in $x$-direction, and minor axis of $20 \mathrm{~mm}$, in both $y$ - and $z$-direction. Their interception with the tube (i.e., the height of the sinus) measures $18.75 \mathrm{~mm}$ and their maximum width is $5.5 \mathrm{~mm}$. Moreover, their position is radial-symmetric in the plane orthogonal to the streamwise direction.

\subsection{Mesh generation}

Different grids were created for the five flow simulations. RANS and "laminar" simulations were performed on the same mesh (Fig. 1c, d, e), based on the Taylor microscale $\lambda$, defined as:

$\lambda=D\left(\frac{10}{R e}\right)^{1 / 2}$

where $R e$ is the Reynolds number, based on average inlet velocity at systolic peak and the tube diameter. This grid size resulted from a convergence study, where three meshes were compared via grid convergence index (GCI) method (Roache, 1997). This analysis assured that the results of the RANS simulations were independent on grid size and that the observed differences in the flow field solutions were due to the intrinsic nature of the turbulence modelling approach. The boundary layer was discretized with a first cell size of $y^{+}=1$, a grow rate of 1.15 in the wall-normal direction and a total thickness of $1.33 \mathrm{~mm}$, based on

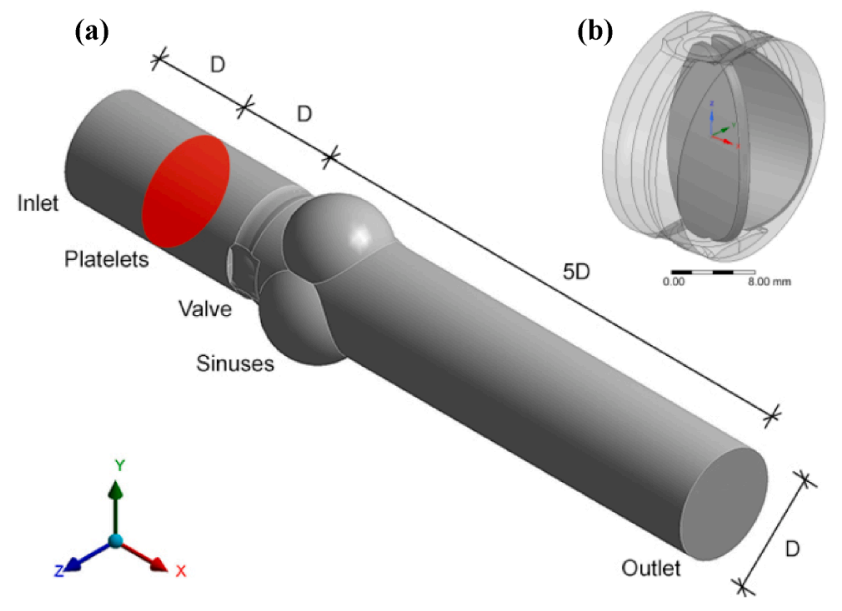

(c)

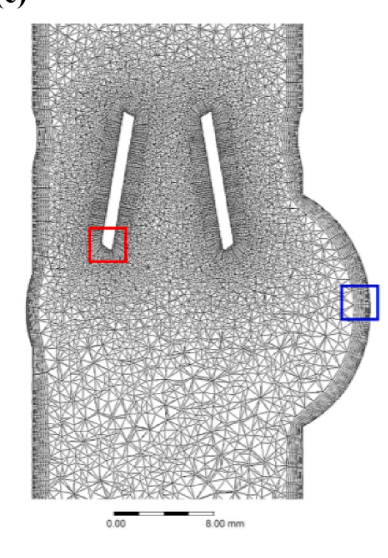

(d)

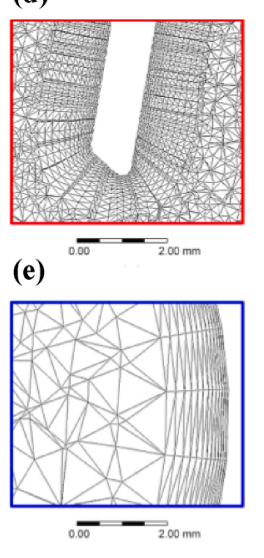

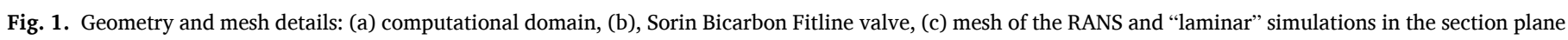
$\mathrm{z}=0$, (d) and (e) closer views of the boundary layer of the leaflets and sinuses, respectively. 
the turbulence intensity.

The LES mesh was generated with a characteristic mesh length of 16 $\eta$ where $\eta$ is the Kolmogorov microscale:

$\eta=D R e^{-3 / 4}$

DNS was performed on a finer mesh with a characteristic size of $8 \eta$, to catch large part of the whole energy cascade (Versteeg and Malalasekera, 2007). The Kolmogorov scale is the smallest scale where turbulent kinetic energy breaks down into heat. Since most of the dissipation takes place at scales larger than $\eta$, the smallest resolved scale can be of order $\eta$ and not exactly $\eta$ (Moin and Mahesh, 1998). In LES and DNS a non-uniform grid resolution was used with the first cell center at $y^{+}=0.1$ and $y^{+}=0.05$ respectively and with a grow rate factor of 1.15 .

The total number of elements resulted in about 3.3 million cells for RANS and "laminar" simulations, 9.3 million for LES and 54 million for DNS. Other DNS studies (Dasi et al., 2007; De Tullio et al., 2009) were much less accurate, with 9.7 and 6.6 million grid points, respectively. The main mesh parameters are reported in Table 1.

\subsection{Numerical approach}

Blood was assumed incompressible and Newtonian with density $\rho=$ $1060 \mathrm{~kg} \mathrm{~m}^{-3}$ and dynamic viscosity $\mu=0.0035 \mathrm{~Pa} \bullet$ s. The flow field was computed from the 3D, unsteady, continuity and momentum equations. ANSYS $®$ Fluent 19.1 software was used for the numerical simulations with SIMPLE algorithm for pressure-velocity coupling and a 2nd order scheme for spatial derivatives. In particular, a least squares cell-based method was used for the gradients and a second order upwind method for pressure and momentum equations. In RANS simulations a 2nd order upwind method was employed for the turbulent kinetic energy $k$ and the specific dissipation rate $\omega$. Default ANSYS ${ }^{\circledR}$ Fluent constants were used for all the turbulent models (SST $k$ - $\omega$, RSM and LES) and a low-Re correction for the SST $k$ - $\omega$ closure. For time derivatives a 1st order implicit formulation was used. All the simulations were performed with a convergence criterion of $10^{-4}$ for the residual errors. The simulations were limited to the systolic phase, i.e. $290 \mathrm{~ms}$. The Eulerian and Lagrangian analysis were limited within the interval between $36 \mathrm{~ms}$ and $231 \mathrm{~ms}$, i.e., the instant of maximum acceleration (A) and deceleration (D) before and after the systolic peak (P) (Fig. 2), respectively. After a sensitivity analysis, a $1 \mathrm{~ms}$ time step was set for RANS and "laminar" simulations, while for LES and DNS $0.25 \mathrm{~ms}$ and $0.1 \mathrm{~ms}$, respectively. A flat velocity profile was imposed at the inlet mimicking an experimental flow rate waveform (Piatti et al. 2015) with peak flow rate of $450 \mathrm{ml} / \mathrm{s}$ (Fig. 2). The resulting inlet Reynolds numbers (based on cross-section mean velocity and tube diameter) were 2500, 7900 and 4000 for the instants of maximum acceleration, systolic peak and maximum deceleration, respectively. This indicates that during the systolic phase the flow regime varies from laminar through transitional to low Re turbulent, with the onset of turbulence at the systolic peak (Ge et al. 2008). A uniform reference pressure equal to zero was prescribed at the outlet. Simulations were run on a HPC cluster equipped with an Intel Xeon E52697 processor with 36 computational cores (clock frequency of 2.30 $\mathrm{GHz}$, shared RAM of $128 \mathrm{~GB}$ ). The CPU time was about $4 \mathrm{~h}$ for RANS simulations, $30 \mathrm{~h}$ for LES and $400 \mathrm{~h}$ for DNS.

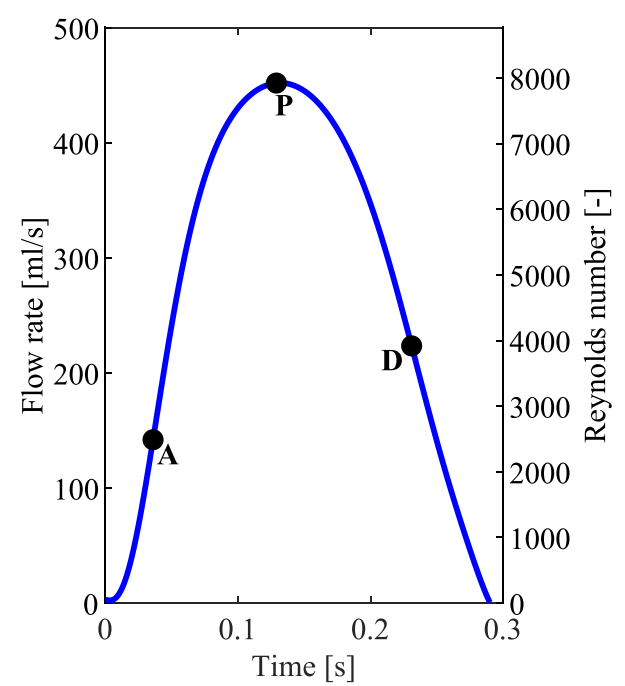

Fig. 2. Inlet flow waveform with relevant time instants: A (maximum acceleration), $\mathrm{P}$ (systolic peak) and $\mathrm{D}$ (maximum deceleration).

\subsection{Platelet injection}

In order to simulate the platelets dynamics, 11,347 neutrally buoyant spherical particles (diameter $3 \mu \mathrm{m}$ ) were simultaneously injected at $t=112 \mathrm{~ms}$, on a $y z$ plane, $1 \mathrm{D}$ upstream the leaflets. The injection time/position were set such that $90 \%$ of the platelets reached the valve (i.e., between $-D / 2$ and $+D / 2$ from the valve) at the systolic peak. A concentric circular seeding pattern was used to compute fluid stresses accurately (Marom and Bluestein, 2016). Particles trajectories were extracted between $-D / 2$ and $+3 D$ to limit the influence of the boundary conditions.

\subsection{Lagrangian analysis and PAS estimation}

The nine components of the stress tensor:

$\tau_{i j}=-\mu\left(\frac{\partial u_{i}}{\partial x_{j}}+\frac{\partial u_{j}}{\partial x_{i}}\right)$

were reduced to a single scalar value:

$\sigma=\left(\frac{1}{12}\left(\tau_{i i}-\tau_{j j}\right)^{2}+\frac{1}{2} \tau_{i j}^{2}\right)^{1 / 2}$

according to Bludszuweit (1994) and Faghih and Sharp (2016), where $\sigma$ is the scalar total stress, which does not depend on the diagonal terms. For the three turbulent closures (SST $k$ - $\omega$, RSM and LES) only the viscous stress components were taken into account. Depending on the turbulence model the off-diagonal components were calculated as follows:

SST $k-\omega: \quad \tau_{i j}=\left(\mu+\mu_{t}\right)\left(\frac{\partial\left\langle u_{i}\right\rangle}{\partial x_{j}}+\frac{\partial\left\langle u_{j}\right\rangle}{\partial x_{i}}\right)$

Table 1

Mesh parameters: number of cells $(\mathrm{N})$, minimum cell volume $\left(\mathrm{V}_{\min }\right)$, maximum cell volume $\left(\mathrm{V}_{\max }\right)$, maximum aspect ratio $\left(\mathrm{AR}_{\max }\right)$, maximum skewness $\left(\mathrm{SK}_{\max }\right)$, minimum ortogonality quality $\left(\mathrm{OQ}_{\mathrm{min}}\right)$, percentage of cells with aspect ratio greater than $5\left(\mathrm{~N}_{\mathrm{AR}}\right)$, skewness above $0.95\left(\mathrm{~N}_{\mathrm{SK}}\right)$ or orthogonal quality less than 0.01 $\left(\mathrm{N}_{\mathrm{OQ}}\right)$.

\begin{tabular}{|c|c|c|c|c|c|c|c|c|c|}
\hline & $\mathrm{N}$ & $\mathrm{V}_{\min }\left[\mathrm{mm}^{3}\right]$ & $\mathrm{V}_{\max }\left[\mathrm{mm}^{3}\right]$ & $\mathrm{AR}_{\max }$ & $\mathrm{SK}_{\max }$ & $\mathrm{OQ}_{\min }$ & $\mathrm{N}_{\mathrm{AR}>5}$ & $\mathrm{~N}_{\mathrm{SK}>0.95}$ & $\mathrm{~N}_{\mathrm{OQ}<0.01}$ \\
\hline $\begin{array}{l}\text { SST } k-\omega \\
\text { RSM } \\
\text { Laminar }\end{array}$ & $3,281,493$ & $3.2^{*} 10^{-5}$ & $7.2^{*} 10^{-2}$ & 37.6 & 0.975 & 0.026 & $0.10 \%$ & $\ll 0.01 \%$ & $0 \%$ \\
\hline LES & $9,263,994$ & $2.9^{*} 10^{-5}$ & $2.2 * 10^{-2}$ & 35.4 & 0.98 & 0.02 & $0.04 \%$ & $\ll 0.01 \%$ & $0 \%$ \\
\hline DNS & $54,397,036$ & $2.6^{*} 10^{-5}$ & $1.3^{*} 10^{-2}$ & 32 & 0.98 & 0.02 & $0.06 \%$ & $\ll 0.01 \%$ & $0 \%$ \\
\hline
\end{tabular}


$\operatorname{RSM}: \quad \tau_{i j}=\mu\left(\frac{\partial\left\langle u_{i}\right\rangle}{\partial x_{j}}+\frac{\partial\left\langle u_{j}\right\rangle}{\partial x_{i}}\right)-\rho\left\langle u_{i} u_{j}\right\rangle$

LES : $\quad \tau_{i j}=\left(\mu+\mu_{t}\right)\left(\frac{\partial \widehat{u}_{i}}{\partial x_{j}}+\frac{\partial \widehat{u}_{j}}{\partial x_{i}}\right)$

DNS and Laminar : $\tau_{i j}=\mu\left(\frac{\partial u_{i}}{\partial x_{j}}+\frac{\partial u_{j}}{\partial x_{i}}\right)$

where $\mu$ and $\mu_{t}$ are the dynamic and turbulent viscosity, $\left\langle u_{i}\right\rangle$ is the timeaverage of $u_{i}$ and $\widehat{u}_{i}$ is the filtered velocity $u_{i}$. In the RSM the modelled quantities are $\mu_{t}$ and the Reynolds stresses $\rho\left\langle u_{i}^{\prime} u_{j}^{\prime}\right\rangle$.

The scalar stress was extracted for each time step along the particle trajectories to determine the stress history of the platelets $\sigma(t)$, which was used to calculate Lagrangian statistics and platelet activation state. Each particle trajectory was characterized by a value of stress accumulation $(S A)$ and stress rate $(S R)$. The former is the integral of the scalar stress over time, calculated as the summation over all the time steps of the product between the scalar stress $\sigma(t)$ and the exposure time. The latter is the variation of the scalar stress over time, calculated as the average over all the time steps of the absolute values of time-derivative of the scalar stress $\sigma(t)$.

The scalar stress history along the trajectories $\sigma(t)$ was also used as input to a phenomenological model of shear-induced platelet activation, which describes the relationship between the history of stress of the platelets and their activation level, called platelet activation state (PAS). The latter is a non-dimensional and continuous measure, defined in the interval $0-1$, where 0 indicate quiescent platelets and 1 fully activated platelets. The model has been successfully validated by Soares et al. (2013) and Consolo et al. (2017), by comparing experimental and numerical predictions of platelet activation in response to different timeconstant and dynamic shear stress conditions. According to the PAS model, the current rate of activation of each platelet is calculated based on the following equation:

$\frac{d P A S(t)}{d t}=K_{0}\left[P A S, \sigma^{(t)}(s)\right](1-P A S)$

where $\sigma^{(t)}(s)=\sigma(t-s), s \in[0, t]$ is the scalar stress history up to time $t, K_{0}$ is the rate of stress-induced platelet activation when PAS $=0$ and the term within square brackets accounts for different phenomena driving the process of shear-mediated platelet activation (described in detailed in the Supplementary Information). Noteworthy, different turbulent models result in different stress tensors (equations from 5 to 8), implying different scalar stress histories and ultimately platelet activation states.

Finally, we calculated the platelet residence time inside the domain (RT), i.e., trajectory duration, and the average curvature $(K)$. This latter is an indicator of flow tortuosity (Braun et al., 2006; Xu et al., 2007) which is relevant in platelet activation (Chesnutt and Han, 2011). As in Braun et al. (2006), the curvature definition used is:

$\kappa=\frac{|\mathbf{u} \times \dot{\mathbf{u}}|}{|\mathbf{u}|^{3}}$

where $\mathbf{u}$ is the velocity vector and $\dot{\mathbf{u}}$ its material derivative. For each particle, at each time step, the instantaneous curvature $\kappa$ has been computed discretizing equation (10) by centered-differences and then the average curvature of each trajectory $K$ has been obtained by taking the mean of the instantaneous values.

\section{Results}

\subsection{Eulerian fields}

The simulations for each model were compared in terms of streamwise velocity profiles at systolic peak (Supplementary Fig. S1) and velocity and scalar stress contours at the three time instants A (Supplementary Fig. S2 and S3), P (Figs. 3 and 4) and D (Supplementary Fig. S5 and S6). In simulations with turbulent closures (SST $k-\omega$, RSM and LES), the scalar viscous stress was also analysed (Supplementary Fig. S4, Fig. 5 and Supplementary Fig. S7 for the instants A, P and D, respectively).

The acceleration phase (A) is mostly laminar and all models perform well (Supplementary Fig. S2 and S3). However, at systolic peak the flow downstream the valve "explodes" in complex and smaller structures (Fig. 3), which are caught only by scale-resolving simulations (LES and DNS). Differences emerge downstream the leaflets where boundary layer separation results in turbulence (Supplementary Fig. S1, from (c) to $(f)$ ), whereas the upstream velocity profile is almost identical for all the simulations (Supplementary Fig. S1 $(a)$ and $(b)$ ). The scalar stress contours (Fig. 4) show that the highest stresses occur at the leaflets, in the wake and in the sinuses. Turbulence model affects stresses more than velocities, as expected, due to their derivative relationship. Differences emerges even among the RANS approaches: RSM results are close to DNS, while the SST $k$ - $\omega$ overestimates the stresses by one order of magnitude. Integral quantities are much closer for all the turbulence models: at the systolic peak the maximum streamwise velocity near the valve ranges between 1.92 and $1.98 \mathrm{~m} / \mathrm{s}$ and the pressure drop varies from 600 to $673 \mathrm{~Pa}$, depending on the simulation (Table 2). This is as expected, since mean flow properties are correctly estimated both by closures based on the Reynolds decomposition (Versteeg and Malalasekera, 2007) and laminar models (Halevi et al., 2016). During the deceleration phase, the flow past the valve exhibits a complex topology induced by a periodic vortex shedding in the wave of the leaflets (Supplementary Fig. S5). An intricate pattern of interacting vortices extending downstream the valve is predicted by both DNS and LES, while the other closures cannot capture the structure of the flow. Fluid stresses are lower than during the deceleration phase but show a more complex topology (Supplementary Fig. S6). Notably, scalar stresses are strongly overestimated not only by the SST $k$ - $\omega$ model but also by the RSM.

\subsection{Lagrangian statistics}

In Fig. 6 the main quantiles of the empirical probability density functions (pdfs) of the Lagrangian variables are reported: platelet activation state $(P A S)$, stress accumulation $(S A)$, stress rate $(S R)$, maximum scalar stress $\left(\sigma_{\max }\right)$. The full distributions are in the Supplementary Fig. S8, S9, S10, S11, S12, S13, respectively). Table 3 contains the relative errors of the mean values, with respect to DNS, considered as the most accurate solution of the flow field.

All turbulence models underestimate PAS with respect to DNS, particularly for higher quantiles. The mean PAS is about 0.008 for DNS and ranges from 0.003 to 0.007 in the other simulations, while the 90 th percentile is 0.017 for DNS and between 0.006 and 0.012 in the other models. The SST $k$ - $\omega$ model and the LES provide the closest results to DNS, with mean and 90th percentile about $10 \%$ and $30 \%$ lower than DNS, respectively. The RSM and the "laminar" simulations estimate a mean and 90th percentiles of PAS much lower than DNS (50\% and 60\%, respectively).

The empirical distributions of SA indicate that the SST $k$ - $\omega$ strongly overestimates (about four-times) the fluid stresses along the trajectories. The RSM and LES also predict higher SA than DNS, with mean values about $50 \%$ and $15 \%$ higher, respectively. Differently, the "laminar" model underestimates the fluid stresses by about $25 \%$ with respect to DNS. The maximum scalar stress is well predicted by the RSM and LES models with errors lower than $10 \%$ for both the mean values and the 90th percentiles. The SST $k$ - $\omega$ largely overestimates by at least twofold all the quantiles of $\sigma_{\max }$ while the "laminar" model predicts mean values about 30\% lower than DNS.

The pdfs of the stress rate closely resemble those of PAS, as expected, given that the shear-loading rate is the most important variable for 
SST

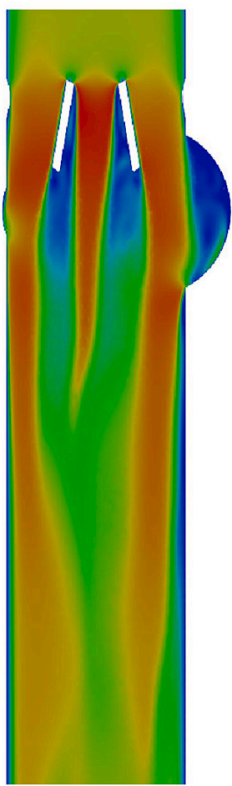

RSM

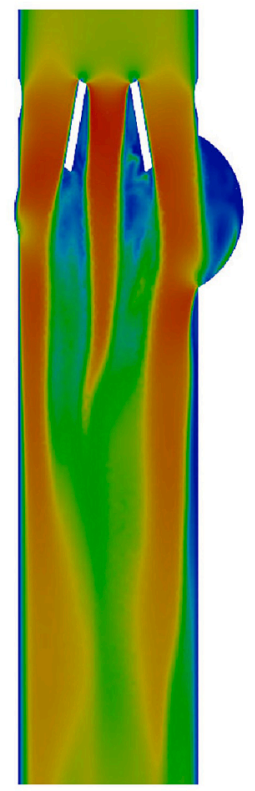

LAM

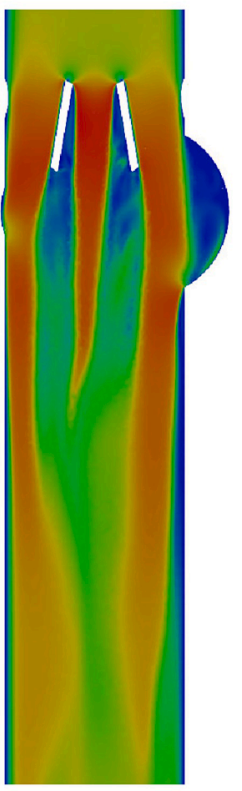

LES

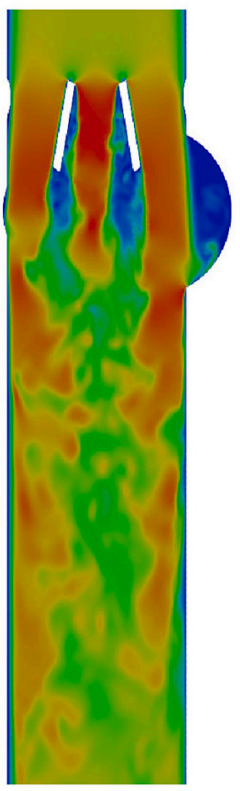

\section{DNS}

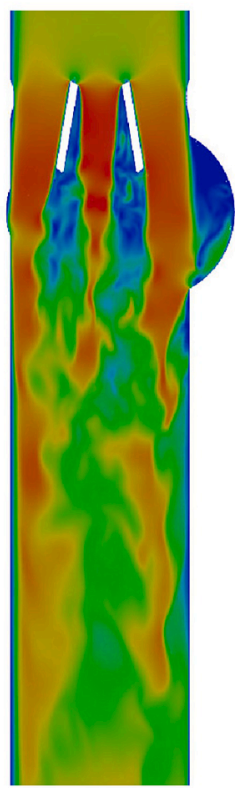

Velocity

0.00

0.50

1.00

1.50

2.00

Fig. 3. Velocity contours on the $\mathrm{z}=0$ plane at the systolic peak.

SST

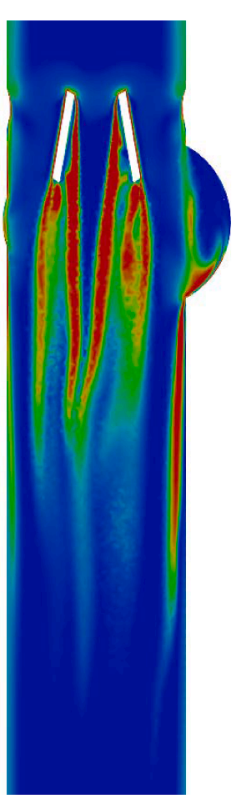

LAM

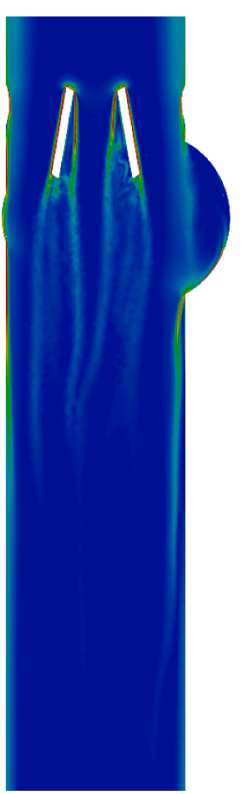

LES

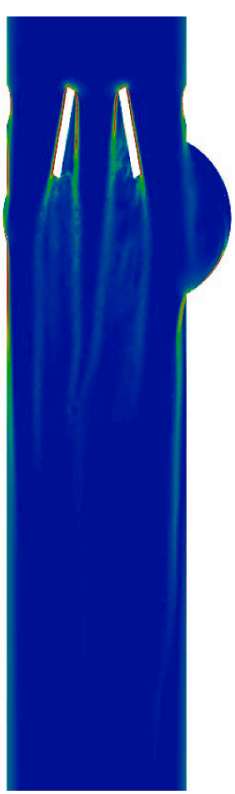

DNS

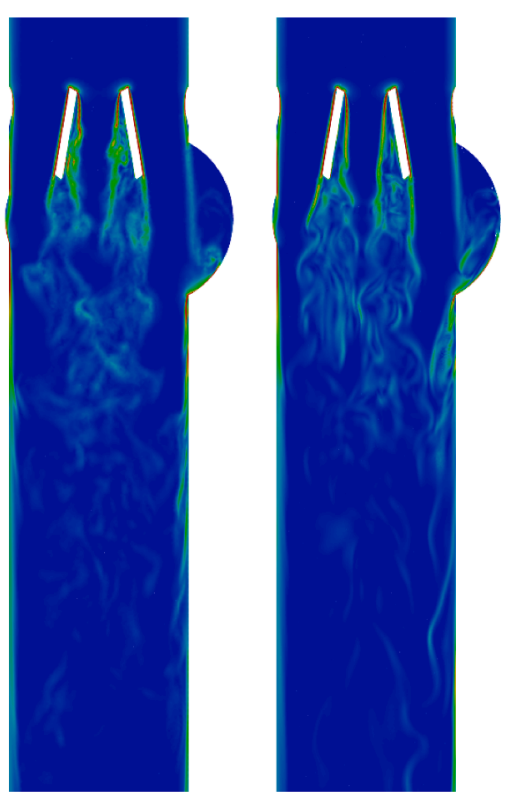

Scalar total stress

[Pa]

10.00

15.00

20.00

Fig. 4. Scalar total stress contours on the $\mathrm{z}=0$ plane at the systolic peak.

platelet activation. $S R$ is in general underestimated by all models, SST $k$ $\omega$ being the closest to DNS followed by LES, RSM and "laminar". The differences, with respect to DNS, range from $1 \%$ to $71 \%$ for the mean values and from $21 \%$ to $74 \%$ for the 90 th percentiles.

\section{Discussion}

Lagrangian statistics offers physically consistent metrics for different blood damages: hemolysis, platelet activation and von Willebrand disease (Kim et al. 2019; Yu et al. 2017). In last decades, this approach has been successfully used to design and optimize different blood-contacting devices, including VAD, valves and oxygenators. However, blood 
SST

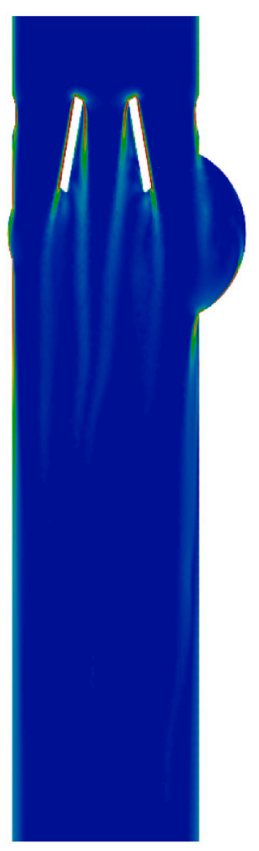

Scalar viscous stress [Pa]
RSM

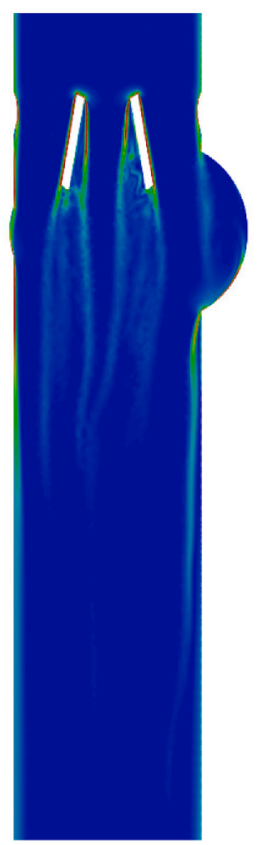

LES

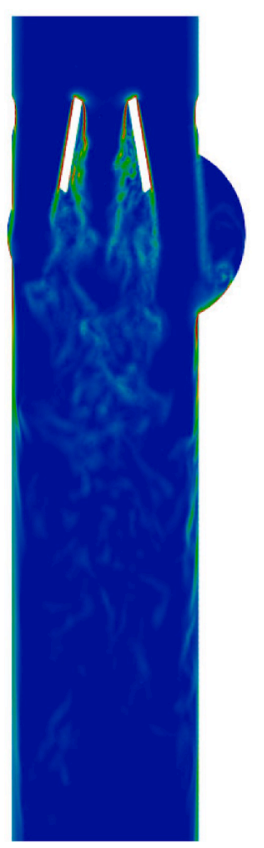

20.00
Fig. 5. Scalar viscous contours on the $\mathrm{z}=0$ plane at the systolic peak.

Table 2

Pressure drop through the valve and maximum streamwise velocity at the systolic peak.

\begin{tabular}{llllll}
\hline & SST $k-\omega$ & RSM & Laminar & LES & DNS \\
\hline$\Delta \mathrm{p}[\mathrm{Pa}]$ & 651 & 673 & 640 & 601 & 600 \\
$u_{\max }[\mathrm{m} / \mathrm{s}]$ & 1.96 & 1.92 & 1.94 & 1.98 & 1.94 \\
\hline
\end{tabular}

damage models rely on the flow field, namely on shear stresses, i.e., on local velocity gradients. As such, reliable blood damage predictions are only feasible when the hemodynamics of the devices is accurately solved. This is fairly straightforward for laminar flows, but it is challenging in the transitional regime (Bhushan et al., 2013; Fraser et al., 2012; Malinauskas et al., 2017). As highlighted by the FDA Critical Path Initiative, when cardiovascular flows range from laminar through transitional to low turbulent, no turbulence model can be considered consistent and accurate (Bhushan et al., 2013). Moreover, in Reynoldsbased turbulence models the shear stress is not uniquely expressed thus leading to controversial results with different blood damage models (Goubergrits et al., 2019; Ge et al., 2008); while in "laminar" and in DNS simulations the shear stress actually denotes a physical force, in turbulent closures it is all but a real quantity, rather being a statistical quantity of the velocity field with a weak physical meaning.

For a specific MHV and PAS model (Consolo et al., 2017; Soares et al., 2013), here we investigated to which extent the Lagrangian assessment of platelet activation depends on the turbulent closure.

The velocity fields obtained from the SST $k$ - $\omega$ and RSM turbulent models strongly depart from the DNS solution, here assumed as the gold standard, and even from the accurate LES model. Differences and physical inconsistences amplify when considering derivatives of the velocity, such as the viscous and Reynolds stresses, as already pointed out by Ge et al. (2008). In the SST $k$ - $\omega$ simulation the scalar stress field results in much higher values than the RSM and the "laminar" scheme and extremely different from the more consistent LES and DNS.

Platelet activation may then vary up to $60 \%$ depending on the

Table 3

Relative errors of the turbulence models with respect to DNS, reported as mean over all the platelets (All) and mean over the platelets with PAS above the 90th percentile (Tail).

\begin{tabular}{llllll}
\hline \multirow{2}{*}{ PAS } & & SST $\boldsymbol{k}$ - $\omega$ & RSM & Laminar & LES \\
& All & $-8 \%$ & $-57 \%$ & $-62 \%$ & $-16 \%$ \\
SA & Tail & $1 \%$ & $-55 \%$ & $-61 \%$ & $-13 \%$ \\
& All & $291 \%$ & $54 \%$ & $-25 \%$ & $15 \%$ \\
SR & Tail & $315 \%$ & $65 \%$ & $-24 \%$ & $19 \%$ \\
& All & $1 \%$ & $-63 \%$ & $-71 \%$ & $-19 \%$ \\
$\sigma_{\max }$ & Tail & $9 \%$ & $-62 \%$ & $-71 \%$ & $-17 \%$ \\
\multirow{3}{*}{$\mathbf{R T}$} & All & $155 \%$ & $-5 \%$ & $-33 \%$ & $10 \%$ \\
& Tail & $198 \%$ & $3 \%$ & $-34 \%$ & $12 \%$ \\
$\log (\mathbf{K})$ & All & $-1 \%$ & $-4 \%$ & $-5 \%$ & $4 \%$ \\
& Tail & $-2 \%$ & $-4 \%$ & $-5 \%$ & $4 \%$ \\
& All & $-30 \%$ & $-20 \%$ & $-20 \%$ & $9 \%$ \\
& Tail & $-30 \%$ & $-20 \%$ & $-20 \%$ & $9 \%$ \\
\hline
\end{tabular}
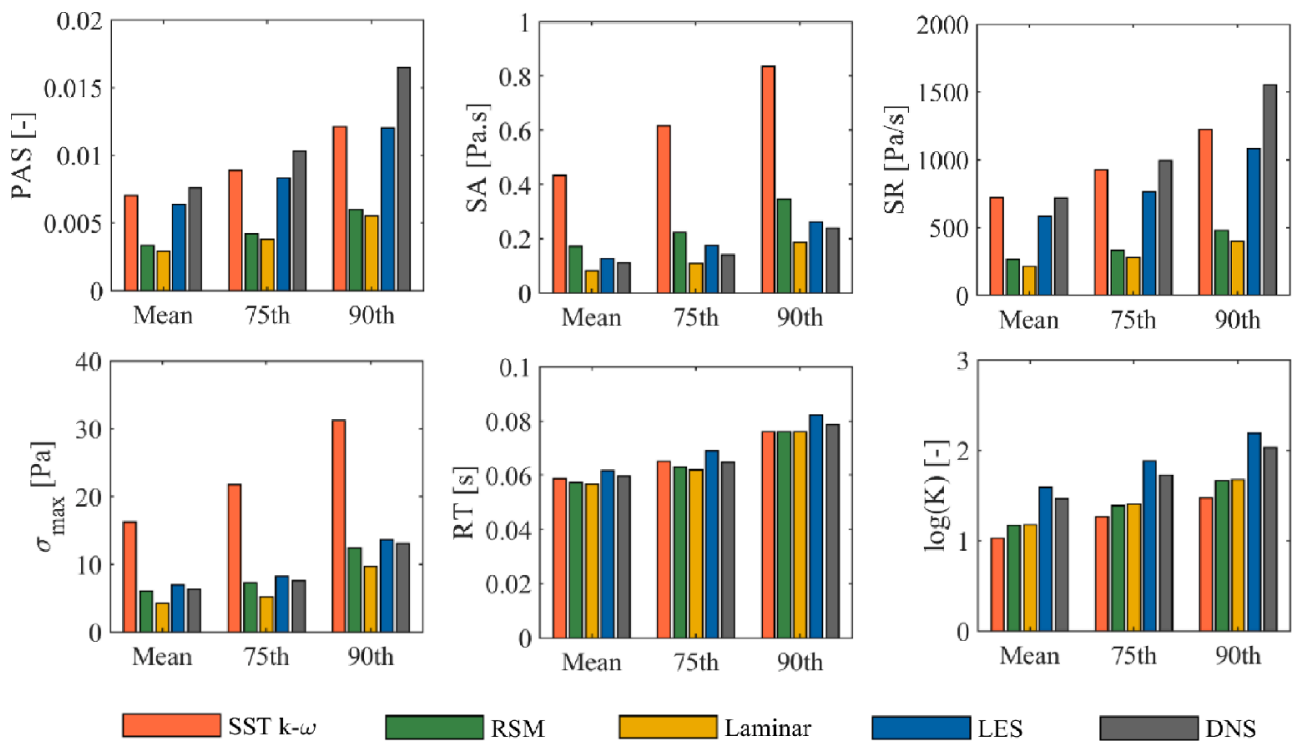

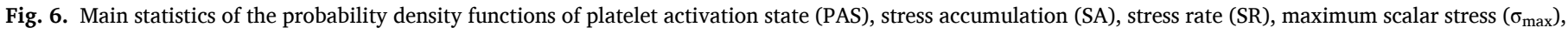
residence time (RT) and trajectory curvature (K). 
turbulent closure, with the exception of SST $k$ - $\omega$. However, in this case, the statistical distributions of shear stress and shear rate are inconsistent, and it is likely an incidental combination that SST $k$ - $\omega$ and DNS results are similar in terms of PAS. Indeed, the SST $k$ - $\omega$ greatly overestimates the shear stress (and shear rate) even if velocities are comparable with other closures. Importantly, all the turbulent closures underestimate the platelet activation with respect to DNS.

The statistical distributions of the curvature (Supplementary) indicate that trajectories are shorter in filtered simulations (SST $k-\omega$, RSM, "laminar"). The formal structure of RANS models hampers to capture the small and fast evolving flow structures, which are essential to accurately estimate the trajectories and the related Lagrangian variables.

The scalar stress analysis (Fig. 4) confirms that the SST $k$ - $\omega$ model overestimates the shear stresses acting on the platelets, especially near the valve, where the scalar total stress is much higher than the viscous one, i.e., the contribution of Reynolds stresses overwhelms that of laminar ones. On the other hand, the "laminar" simulation on a coarser grid leads to an underestimation of the fluid stresses because the viscous stresses are insufficiently resolved.

From the statistical distribution and quantiles of the stress rate emerges that it is larger in DNS and LES than in RSM. This is due to the fact that scale-resolving simulations can catch the variability of the stresses more accurately than Reynolds-averaged models. Moreover, the "laminar" model gives the lowest $S R$ values showing that the Taylorbased mesh is not enough to resolve the small scale structures; this occurrence, summed up to the lack of a $S R$ model, leads to a large underestimation of $S R$.

According to our results, the Lagrangian analysis can be highly altered when adopting a turbulence model with respect to DNS, which accounts for all the relevant flow structures. Indeed, turbulence models unavoidable when reduction of computational cost is a practical need imply the straightening of the trajectories; hence, to be consistent with the real energy dissipation, the artificial turbulent shear stress, is necessarily added. In this way the flow energy dissipation matches the experimental observations. Subgrid turbulence modelling used in the LES approach, also affects the Lagrangian analysis, although to a minor extent. Indeed, the LES local flow structures are quite similar to the DNS ones.

Within the limits of the aforementioned assumptions, from our work emerge that DNS and, within an acceptable approximation, LES should be used when assessing blood cell damage by means of coupled EulerianLagrangian CFD simulations. Conversely, RANS turbulent closures should be avoided when predicting platelet activation in turbulent and transitional flows through Lagrangian statistics.

In a more general perspective, new Lagrangian-consistent turbulent models are necessary, because DNS and LES are not always feasible in terms of computational costs.

\section{Declaration of Competing Interest}

The authors declare that they have no known competing financial interests or personal relationships that could have appeared to influence the work reported in this paper.

\section{Appendix A. Supplementary material}

Supplementary data to this article can be found online at https://doi. org/10.1016/j.jbiomech.2021.110704.

\section{References}

Ando, J., Yamamoto, K., 2009. Vascular mechanobiology: endothelial cell responses to fluid shear stress. Circ. J. 73, 1983-1992.

Bhushan, S., Walters, D.K., Burgreen, G.W., 2013. Laminar, turbulent, and transitional simulations in benchmark cases with cardiovascular device features. Cardiovasc. Eng. Tech. https://doi.org/10.1007/s13239-013-0155-5.
Bludszuweit, C., 1994. A Theoretical Approach to the Prediction of Haemolysis in Centrifugal Blood Pumps. PhD, thesis. University of Strathclyde, Glasgow.

Braun, W., De Lillo, F., Eckhardt, B., 2006. Geometry of particle paths in turbulent flows. J. Turb. https://doi.org/10.1080/14685240600860923.

Casa, L., Deaton, D., Ku, D., 2015. Role of high shear rate in thrombosis. J. Vasc. Surg. $61,1068-1080$.

Cheng, R., Lai, Y., Chandran, K., 2004. Three-dimensional fluid-structure interaction simulation of bileaflet mechanical heart valve flow dynamics. Ann. Biomed. Eng. 32 (11), 1471-1483.

Chesnutt, J.K.W., Han, H.C., 2011. Tortuosity triggers platelet activation and thrombus formation in microvessels. J. Biomech. Eng. 133, 121004.

Consolo, F., Sheriff, J., Gorla, S., Magri, N., Bluestein, D., Pappalardo, F., et al., 2017. High frequency components of hemodynamic shear stress profiles are a major determinant of shear-mediated platelet activation in therapeutic blood recirculating devices. Sci. Rep. 7, 4994.

Consolo, F., Sferrazza, G., Motolone, G., Pieri, M., De Bonis, M., Zangrillo, A., et al., 2019. Shear-mediated platelet activation enhances thrombotic complications in patients with LVADs and is reversed after heart transplantation. ASAIO J. 65, e33-e35.

Dasi, L., Ge, L., Simon, H., Sotiropoulos, F., Yoganathan, P., 2007. Vorticity dynamics of a bileaflet mechanical heart valve in an axisymmetric aorta. Phys. Fluids 19, 067105.

De Tullio, M., Cristallo, A., Balaras, E., Verzicco, R., 2009. Direct numerical simulation of the pulsatile flow through an aortic bileaflet mechanical heart valve. J. Fluid Mech. 622, 259-290.

Faghih, M., Sharp, M., 2016. Extending the power-law hemolysis model to complex flows. J. Biomech. Eng. 138, 124504.

Fraser, K.H., Zhang, T., Taskin, M.E., Griffith, B.P., Wu, Z.J., 2012. A quantitative comparison of mechanical blood damage parameters in rotary ventricular assist devices: shear stress, exposure time and hemolysis index. J. Biomech. Eng. 134 081002 .

Ge, L., Dasi, L.P., Sotiropoulos, F., Yoganathan, A.P., 2008. Characterization of hemodynamic forces induced by mechanical heart valves: Reynolds vs. viscous stresses. Ann. Biomed. Eng. 36, 276-297.

Giersiepen, M., Wurzinger, L., Opitz, R., Reul, H., 1990. Estimation of shear stress-related blood damage in heart valve prostheses in vitro comparison of 25 aortic valves. Int. J. Artif. Organs 13, 300-306.

Goubergrits, L., Hellmeier, F., Neumann, D., Mihalef, V., Gulsun, M., Chinali, M., et al., 2019. Patient-specific requirements and clinical validation of MRI-based pressure mapping: a two-center study in patients with aortic coarctation. JMRI 49, 81-89.

Halevi, R., Hamdan, A., Marom, G., Lavon, K., Ben-Zekry, S., Raanani, E., et al. Fluid-structure interaction modeling of calcific aortic valve disease using patient-specific three-dimensional calcification scans. Med. Biol. Eng. Comput. 54, 1683-1694.

Kroll, M., Hellums, J., McIntire, L., Schafer, A., Moake, J., 1996. Platelets and shear stress. Blood 88, 1525-1541.

Kim, D., Bresette, C., Liu, Z., Kua, D.N., 2019. Occlusive thrombosis in arteries. API Bioeng. 3, 041502.

Ku, D., 1997. Blood flow in arteries. Ann. Rev. Fluid Mech. 29, 399-434.

Le, T., Sotiropoulos, F., 2013. Fluid-structure interaction of an aortic heart valve prosthesis driven by an animated anatomic left ventricle. J. Comput. Phys. 244 41-62.

Malinauskas, R., Hariharan, P., Day, S., Herbertson, L., Buesen, M., Steinseifer, U., et al., 2017. FDA benchmark medical device flow models for CFD validation. ASAIO J. 63, $150-160$.

Marom, G., Bluestein, D., 2016. Lagrangian methods for blood damage estimation in cardiovascular devices - how numerical implementation affects the results. Expert Rev. Med. Devices 13, 113-122.

Moin, P., Mahesh, K., 1998. Direct numerical simulation: a tool in turbulence research. Ann. Rev. Fluid Mech. 30, 539-578.

Morbiducci, U., Ponzini, R., Nobili, M., Massai, D., Montevecchi, F.M., Bluestein, D., Redaelli, A., 2009. Blood damage safety of prosthetic heart valves. Shear-induced platelet activation and local flow dynamics: a fluid-structure interaction approach. J. Biomech. 42, 1952-1960.

Nesbitt, W., Westein, E., Tovar-Lopez, F., Tolouei, E., Mitchell, A., Fu, J., et al., 2009. A shear gradient-dependent platelet aggregation mechanism drives thrombus formation. Nat. Med. 15, 665-673.

Nobili, M., Sheriff, J., Morbiducci, U., Redaelli, A., Bluestein, D., 2008. Platelet activation due to hemodynamic shear stresses: damage accumulation model and comparison to in vitro measurements. ASAIO J. 54, 64-72.

Pal, A., Anupindi, K., Delorme, Y., Ghaisas, N., Shetty, D.A., Frankel, S.H., 2014. Large eddy simulation of transitional flow in an idealized stenotic blood vessel: evaluation of subgrid scale models. J. Biomech. Eng. 136, 071009.

Peura, J., Colvin-Adams, M., Francis, G., Grady, K., Hoffman, T., Jessup, M., et al., 2012. Recommendations for the use of mechanical circulatory support: device strategies and patient selection: a scientific statement from the American Heart Association. Circulation 126, 2648-2667.

Piatti, F., Sturla, F., Marom, G., Sheriff, J., Claiborne, T.E., Slepian, M.J., et al., 2015. Hemodynamic and thrombogenic analysis of a trileaflet polymeric valve using a fluid-structure interaction approach. J. Biomech. 48, 3641-3649.

Prandtl, L., 1925. Bericht uber untersuchung zur ausgebildeten turbulenz. Z. Angew. Math. Mech. 5, 136-139.

Roache, P.J., 1997. Quantification of uncertainty in computational fluid dynamcis. Ann. Rev. Fluid Mech. 29, 123-160.

Ruggeri, Z., Orje, J., Habermann, R., Federici, A., Reininger, A., 2006. Activationindependent platelet adhesion and aggregation under elevated shear stress. Blood 108, 1903-1910. 
Russell-Puleri, S., Dela Paz, N., Adams, D., Chattopadhyay, M., Cancel, L., Ebong, E., et al., 2017. Fluid shear stress induces upregulation of COX-2 and PGI2 release in endothelial cells via a pathway involving PECAM-1, PI3K, FAK, and p38. Am. J. Physiol. Heart Circ. Physiol. 312, H485-H500.

Slepian, M., Sheriff, J., Hutchinson, M., Tran, P., Bajaj, N., Garcia, J., et al., 2017. Shearmediated platelet activation in the free flow: perspectives on the emerging spectrum of cell mechanobiological mechanisms mediating cardiovascular implant thrombosis. J. Biomech. 50, 20-25.

Soares, J., Sheriff, J., Bluestein, D., 2013. A novel mathematical model of activation and sensitization of platelets subjected to dynamic stress histories. Biomech. Model. Mechanobiol. 12, 1127-1141.
Song, X., Throckmorton, A., Wood, H., Antaki, J., 2003. Computational fluid dynamics prediction of blood damage in a centrifugal pump. Artif. Organs 27, 938-941.

Versteeg, H.K., Malalasekera, W., 2007. An Introduction to Computational Fluid Dynamics: The Finite Volume Method. Pearson, US.

Xu, H., Ouellette, N.T., Bodenschatz, E., 2007. Curvature of Lagrangian trajectories in turbulence. Phys. Rev. Lett. 98, 050201.

Yoganathan, A., Chandran, K., Sotiropoulos, F., 2005. Flow in prosthetic heart valves: state-of-the-art and future directions. Ann. Biomed. Eng. 33, 1689-1694.

Yu, H., Engel, S., Janiga, G., Thévenin, D., 2017. A review of hemolysis prediction models for computational fluid dynamics. Artif. Organs 41, 603-621. 